\title{
Polarization Characteristics of Pulsar Profiles
}

\author{
J.L. $\operatorname{Han}^{1,2}$, R.N. Manchester ${ }^{2}$, G.J. Qia.o ${ }^{3}$ \\ ${ }^{1}$ National Astronomical Observatories, Beijing 100012, China \\ ${ }^{2}$ A ustralia Telescope National Facility, CSIRO, Australia \\ ${ }^{3}$ Department of Astronomy, Peking University, Beijing 100871, China
}

\begin{abstract}
Polarization profiles of several hundred pulsars have been published recently (eg. Gould \& Lyne 1998, GL98; Weisberg et al. 1999). In this report, we summarize the characteristics of circular and high linear polarizations of pulsar profiles, based on all previously published data.
\end{abstract}

\section{Circular Polarization}

Han et al. (1998) systematically studied the circular polarization of pulsar profiles. They found that circular polarization is common in profiles but diverse in nature. Circular polarization is about $9 \%$ in average, weaker than linear polarization $(15 \%)$ in general. We emphasize the following points about circular polarization $(\mathrm{CP})$ :

(1). CP not unique to core emission: One misleading concept is that circular polarization alway accompanies core emission. It is generally strongest in the central or 'core' regions of a profile, but is by no means confined to these regions. Circular polarization has been detected from conal components of many pulsars, for example, conal-double pulsars.

(2). Sense reversal not unique to core: Circular polarization often changes sense near the middle of the profile. But sense reversals have been observed at other longitudes, e.g. conal components of PSRs B0834+06, B1913+16, $\mathrm{B} 2020+28, \mathrm{~B} 1039-19, \mathrm{~J} 1751-4657$, and B0329+54 in abnormal mode.

(3). No PA correlation for core emission: There is no correlation between the sense of the sign change of circular polarization and the sense of variation of linear polarization angle (PA), in contrast to earlier conclusions on this issue. (4). Correlation for cone-dominated pulsars: We found a strong correlation between between the sign of PA variation and sense of circular polarization in conal-double pulsars, with right-hand (negative) circular polarization accompanying increasing PA and vice versa. No good examples contrary to this trend have been found.

(5). Variation with frequency: Circular polarization generally does not vary systematically with frequency. Multifrequency profiles of many pulsars show similar CP across a wide frequency range (eg. PSRs B0329+54, B0525+21). However, we have found several examples with significant variations (Table 5 of Han et al 1998). For example, PSR B1749-28 now has been confirmed by the data of GL98 to change from dominant right hand ("-") CP at low frequencies to a sense change " $-/+$ " at high frequencies, while PSRs B1859+03 and B1900+01 change from "+" to " $+/-$ ". 


\section{High Linear Polarization}

Strong linear polarization is an outstanding characteristics of pulsars. Almost all pulsars with $\log \dot{E}>34.5$, if a polarization profile is available, have (at least) one highly linearly polarized component. Using our profile database, we found that the highly linearly polarized components do not have to be associated with high $\dot{E}$. Several types of pulsar profiles have highly polarized components:

(1). Leading-polarized component: The prototype of this kind of pulsars is PSR B0355+54. The leading component is almost $100 \%$ polarized. The component does not dominate the profile below several hundred $\mathrm{MHz}$, but becomes stronger towards high frequency. It may be emitted from a different region. Other examples are PSRs B0450+55, B1842+14, B2021+51, B0809+74, B0626+24, B1822-09.

(2). Trailing-polarized component: PSR B0559-05 is a mirror-symmetrical type to PSR B0355+54. Its trailing component is highly linearly polarized, and becomes stronger with increasing frequency. Good examples are PSR B2224+65, $\mathrm{J} 1012+5307$ and $\mathrm{J} 1022+1001$. The latter two are millisecond pulsars.

(3). Polarized multicomponents: The prototype PSR B0740-28 has 7 Gaussian components fitted to a high time resolution profile. These pulsars have a sharp leading edge and more gradual trailing edge, and are highly polarized. Good examples are PSRs J0538+2817, B0540+23, B0833-45, B0950+08i, $\mathrm{J} 1359-6038$, B1929+10m. PSRs B0538-75, J0134-2937 and the postcursor of B0823+26 have just mirror-symmetrical profiles to these examples above.

(4). Polarized single component: Almost $100 \%$ polarized single components may be conal emission emitted from the very outer edge of beam. Two dozen examples have been found. The best examples are PSRs B0105+65, B0611+22, B0628-28, B1322+83, B1556-44, J1603-5657, B1706-44, B1828 $-10, \mathrm{~B} 1848+13, \mathrm{~B} 1913+10, \mathrm{~B} 1915+13$.

(5). Interpulse pulsars: PSR B0906-49 and B1259-63 are young, energentic $(\dot{E}>35)$, interpulse pulsars with extremely highly polarization. These may be pulsars where both sides of a wide conal beam from a single pole are observed. PSR J0631+1036 may be an off-centre cut through a wide cone.

The polarization characteristics of the mean pulse profile provide a framework for understanding the emission processes in pulsars. The characteristics of pulsar circular polarization summarized by Han et al. (1998) should be considered by all emission models. The points on highly linear polarization we make here should be included into future pulsar classifications and geometrical studies of pulsar emission beam.

Acknowledgements JLH thanks financial support from the National Natural Science Foundation (NNSF) and the Educational Ministry of China.

\section{References}

Gould D.M., Lyne A.G., 1998, MNRAS 301, 235

Han J.L., Manchester R.N., Xu R.X., Qiao G.J., 1998, MNRAS 300, 373

Weisberg J.M., Cordes J.M., Lundgren S.C., et al., 1999, ApJS 121, 171 